\title{
FUNCTORS OF SUBDESCENT TYPE AND DOMINION THEORY
}

\author{
P. B. JOHNSON
}

(Communicated by Andreas R. Blass)

\begin{abstract}
Necessary and sufficient conditions are given for the EilenbergMoore comparison functor $\Phi$ arising from a functor $U$ (having a left adjoint) to be a Galois connection in the sense of J. R. Isbell, in which case the functor $U$ is said to be of subdescent type. These conditions, when applied to a contravariant hom-functor $U=\mathbf{C}(-, B): C^{\text {op }} \rightarrow$ Set, read like a kind of functional completeness axiom for the object $B$. In order to appreciate this result, it is useful to consider the full subcategory $\operatorname{dom}_{B} \subset \mathrm{C}$ of so-called $B$-dominions, consisting of certain canonically arising regular subobjects of powers of the object $B$. The functor $U=C(-, B)$ is of subdescent type if and only if the object $B$ is a regular cogenerator for the category $\operatorname{dom}_{B}$, in which case $\operatorname{dom}_{B}$ is the reflective hull of $B$ in $C$ and, moreover, the category $\operatorname{dom}_{B}$ admits a Stone-like representation as (being contravariantly equivalent, via the comparison functor $\Phi$, to) a full, reflective subcategory of the category of algebras for the triple in Set induced by the functor $U$.
\end{abstract}

\section{INTRODUCTION}

In [MV] and [J1] it is shown that the category Lind of completely regular Lindelöf locales admits several characterizations of a purely category-theoretical nature which distinguish it amongst full subcategories of the category Loc of all locales. For one, Lind is the reflective hull of the object $\mathbb{R}$ (the locale corresponding to the usual topology on the set of real numbers) in Loc; in addition, Lind is the largest full subcategory of Loc for which the object $\mathbb{R}$ serves as a regular cogenerator [BW]; and finally, the subcategory Lind is comprised of precisely those objects $X \in|\mathbf{L o c}|$ which are uniquely recoverable from the algebraic data $\operatorname{Loc}(X, \mathbb{R})$.

In this paper it is shown that the three phenomena alluded to above, about the object $\mathbb{R} \in|\mathbf{L o c}|$ and the subcategory Lind $\subset$ Loc, are simultaneously detectable via a simple analysis of the descent behaviour of the contravariant hom-functor $\operatorname{Loc}(-, \mathbb{R}):$ Loc $^{\text {op }} \rightarrow$ Set and similarly for any hom-functor $\mathbf{C}(-, B): \mathbf{C}^{\text {op }} \rightarrow$

Received by the editors May 20, 1992 and, in revised form, January 25, 1993; presented by the author in conference with the Kurt Gödel Society, Technical University, Vienna, Austria, on November 18, 1993.

1991 Mathematics Subject Classification. Primary 18C15; Secondary 06A15, 18A32, 18A22.

This paper, consisting primarily of excerpts from the author's $\mathrm{Ph} . \mathrm{D}$. thesis, Wesleyan University, Middletown, Connecticut, 1992, also represents, in part, the results of research engaged in at Charles University, Prague, during the 1992-1993 academic year, with the support of the United States Information Agency and the Fulbright Program. 
Set, assuming that the prerequisite constructions-powers of the object $B$ and equalizers of certain pairs of maps between these powers-exist in the category C. In fact, as suggested in the abstract, the proposed descent analysis is carried out for an arbitrary functor $U$ having a left adjoint.

We begin with the assumption of familiarity with the rudiments of the theory of triples as found in [BW], and all that follows is in reference to a fixed functor $U: \mathbf{X} \rightarrow \mathbf{A}$ having a left adjoint $F$, with counit and unit maps $\varepsilon: F U \rightarrow 1_{\mathbf{X}}$ and $\eta: 1_{\mathbf{A}} \rightarrow U F$, respectively, associated triple $\mathbb{T}$ in the category $\mathbf{A}$, and Eilenberg-Moore factorization through the comparison functor $\Phi$ as depicted in the diagram of categories and functors

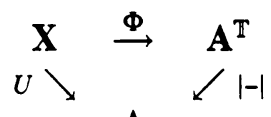

A

where $\mathbf{A}^{\mathrm{T}}$ is the category of triple algebras and $|-|: \mathbf{A}^{\mathrm{T}} \rightarrow \mathbf{A}$ is the functor assigning underlying A-data.

The fundamental result of triple theory, as found in the seminal work of Beck [B], gives necessary and sufficient conditions for the comparison functor to be a full and faithful embedding of the category $\mathbf{X}$ into the category of algebras, in which case the functor $U$ is said to be of descent type. In practice, as we shall see, it is possible that the functor $U$ fails to be of descent type, yet still gives rise to an interesting comparison of the categories $\mathbf{X}$ and $\mathbf{A}^{\mathrm{T}}$. The foundations for this work are contained in the following two lemmas.

1.1. The full isomorphism-closed image of a functor $U: \mathbf{X} \rightarrow \mathbf{A}$ is denoted by $\operatorname{Im}(U) \subset \mathbf{A}$, and we write $\Sigma_{U} \subset \mathbf{X}$ for the subcategory consisting of all those $\mathbf{X}$-morphisms $f$ for which $U f$ is an isomorphism in $\mathbf{A}$.

Lemma. An X-morphism $X \stackrel{f}{\longrightarrow} Y$ satisfies $f \in \Sigma_{\Phi}$ if and only if the induced natural transformation $\mathbf{X}(F-, X) \stackrel{f^{*}}{\longrightarrow} \mathbf{X}(F-, Y)$ is an isomorphism of functors.

Proof. Consider the diagram of categories and functors

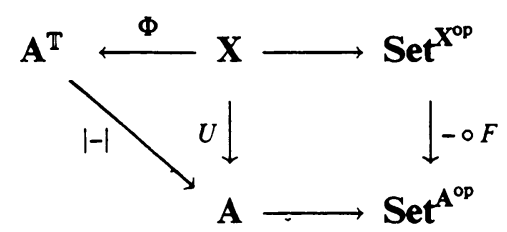

in which the square, depicting the Yoneda embeddings of the categories $\mathbf{X}$ and A, commutes up to natural isomorphism by virtue of $F \dashv U$. The image in Set $^{\mathbf{A}^{\text {op }}}$ of the $\mathbf{X}$-morphism $f$ is readily seen to be $f^{*}$. The result now follows from the fact that both the Yoneda embeddings and the underlying functor $|-|: \mathbf{A}^{\mathbf{T}} \rightarrow \mathbf{A}$ reflect isomorphisms; that is, $f \in \Sigma_{\Phi}$ if and only if $f \in \Sigma_{U}$ if and only if $f^{*}$ is an isomorphism in $\mathbf{S e t}^{\mathbf{A}^{\mathrm{AP}}}$.

1.2. Given $\varepsilon \in \mathbf{X}(Y, E)$, we write $\operatorname{Rel}(\varepsilon)$ to denote the family consisting of all those pairs of maps $(a, b): F A \rightrightarrows Y$, from free objects to $Y$, satisfying 
$\varepsilon a=\varepsilon b$. The diagram

$$
X \underset{g}{\stackrel{f}{\rightrightarrows}} Y \stackrel{\varepsilon}{\rightarrow} E
$$

of X-morphisms is a $U$-absolute coequalizer diagram provided $\varepsilon f=\varepsilon g$ and, for every category $\mathbf{B}$ and functor $H: \mathbf{A} \rightarrow \mathbf{B}$, the diagram $H U(*)$ is a coequalizer in $\mathbf{B}$.

Lemma. Provided the diagram $(*)$ is a $U$-absolute coequalizer diagram, maps $Y \stackrel{t}{\rightarrow} T$ satisfying $t f=t g$ also satisfy $t a=t b$ for every pair $(a, b) \in \operatorname{Rel}(\varepsilon)$.

Proof. By hypothesis and with reference once again to the diagram of 1.1 , the diagram of natural transformations

$$
\mathbf{X}(F-, X) \underset{g^{*}}{\stackrel{f^{*}}{\rightrightarrows}} \mathbf{X}(F-, Y) \stackrel{\varepsilon^{*}}{\rightarrow} \mathbf{X}(F-, E)
$$

is a coequalizer in $\mathbf{S e t}^{\mathbf{A}^{\mathrm{op}}}$. If $t f=t g$, then $\mathbf{X}(F-, Y) \stackrel{t^{*}}{\rightarrow} \mathbf{X}\left(F_{-}, T\right)$ factors through the coequalizer $\varepsilon^{*}$, and the result follows.

1.3. The comparison functor $\Phi$ has a left adjoint $\Phi$ if and only if the category $\mathbf{X}$ has certain coequalizers [L], which we do, indeed, assume to exist. In particular, for each $X \in|\mathbf{X}|$, the diagram

$$
F U F U X \underset{\varepsilon F U X}{\stackrel{F U \varepsilon X}{\rightrightarrows}} F U X \stackrel{\varepsilon X}{\rightarrow} X
$$

is a $U$-absolute coequalizer diagram, and the $\check{\Phi}$-value $\check{\Phi} \Phi X$ may be taken to be the coequalizer of the pair $(F U \varepsilon X, \varepsilon F U X)$, with the counit map $\check{\varepsilon} X$ appearing as the unique factorization of $\varepsilon X$ through the coequalizer

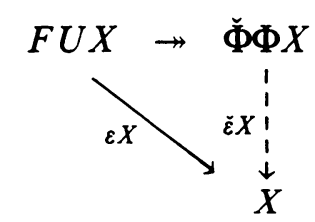

Application of Lemma 1.2 now yields a new description of the counit maps for the adjunction $\check{\Phi} \dashv \Phi$ :

Theorem. Given $X \in|\mathbf{X}|$, the object $\check{\Phi} \Phi X$ is the simultaneous coequalizer of the family $\mathfrak{R e l}(\varepsilon X)$ of all pairs of maps $(a, b): F A \rightrightarrows F U X$, from free objects to $F U X$, satisfying $\varepsilon X \circ a=\varepsilon X \circ b$.

\section{GALOIS CONNECTIONS}

2.1. Given an arbitrary functor $\Phi: \mathbf{X} \rightarrow \mathbf{B}$ having a left adjoint $\check{\Phi}$ with counit and unit maps $\check{\varepsilon}$ and $\check{\eta}$, it is known [12] that the natural transformation $\Phi \check{\varepsilon}: \Phi \check{\Phi} \Phi \rightarrow \Phi$ is an isomorphism if and only if $\check{\Phi} \check{\eta}: \check{\Phi} \rightarrow \check{\Phi} \Phi \check{\Phi}$ is, in which case the functors $\Phi$ and $\check{\Phi}$ restrict to an equivalence of categories $\operatorname{Im}(\check{\Phi}) \cong$ $\operatorname{Im}(\Phi)$ and the original adjunction $\check{\Phi} \dashv \Phi$ (and when there is no possibility of confusion, each of the adjoints $\check{\Phi}$ and $\Phi$ ) is called a Galois connection. To characterize this situation further we mention briefly that $\check{\Phi} \pitchfork$ is a Galois 
connection if and only if the induced cotriple in the category $\mathbf{X}$ (or the induced triple in the category B) is idempotent. Further still, $\check{\Phi} \dashv \Phi$ is a Galois connection if and only the maps $\{\check{\varepsilon} X: \check{\Phi} \Phi X \rightarrow X\}$ constitute a coreflection of $\mathbf{X}$ into $\operatorname{Im}(\check{\Phi})$ or, equivalently, $\check{\eta}$ is a reflection of $\mathbf{B}$ into $\operatorname{Im}(\Phi)$.

When $\Phi$ is the comparison functor arising from the functor $\mathbf{X} \stackrel{U}{\rightarrow} \mathbf{A}$, we say that $U$ is of subdescent type provided $\Phi$ is a Galois connection, and the characterization to follow is the main result of this paper.

Theorem 2.2. $U$ is of subdescent type if and only if, for every $X \in|\mathbf{X}|$, the counit map $\check{\varepsilon} X$ is monomorphic with respect to maps from free objects.

Proof. By Isbell's characterization ( $\$ 2.1), \Phi$ is a Galois connection if and only if $\check{\varepsilon} X \in \Sigma_{\Phi}$, for each $X \in|\mathbf{X}|$. Equivalently, by 1.1, each natural transformation $\mathbf{X}(F-, \breve{\Phi} \Phi X) \stackrel{\check{\varepsilon} X^{*}}{\rightarrow} \mathbf{X}(F-, X)$ is an isomorphism. But each $\check{\varepsilon} X^{*}$ is a fortiori a split epimorphism in Set $^{\mathbf{A}^{\text {op }}}$ (since $\Phi \check{\varepsilon} X$ admits a right inverse $\check{\eta} \Phi X$ in any case). Hence $\check{\varepsilon} X^{*}$ is an isomorphism if and only if it is injective at the level of sets.

\section{Application to sets}

3.1. In the interest of application of the results 1.3 and 2.2 to set-valued functors it is our tendency to prefer the contravariant case. It is well known that a functor $U: \mathbf{C}^{\mathrm{op}} \rightarrow$ Set has a left adjoint $F$ if and only if it is representable, say $U \cong \mathbf{C}(-, B)$, for some object $B$ for which the category $\mathbf{C}$ has all setindexed powers, in which case $F$ may be given, taking as values precisely these powers: $F n=B^{n}$, for each $n \in \mid$ Set $\mid$, with the familiar evaluation maps $e v_{X}: X \rightarrow B^{C(X, B)}$, for each object $X \in|C|$, serving as the counit maps for the adjunction. Writing $\mathbb{B}$ to denote the induced triple, the comparison functor $\Phi_{B}$ appearing in the diagram

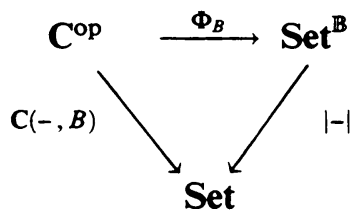

assigns to each object $X \in|C|$ the usual algebra structure on the set $\left|\Phi_{B}(X)\right|=$ $\mathrm{C}(X, B)$.

It is traditional to call the object $B$ a regular cogenerator for the category $\mathbf{C}$ provided that, for each object $X \in|\mathrm{C}|$, the evaluation map $e v_{X}$ is a regular monomorphism or, equivalently, that the comparison functor $\Phi_{B}$ is full and faithful, which is to say, that the functor $U$ is of descent type. In lieu of this very special situation (as suggested in the introduction) it is often possible to extract from the ambient category $\mathbf{C}$ a full subcategory, admitting a nice representation in $\mathbf{S e t}^{\mathbf{B}}$ and for which the object $B$ serves as a regular cogenerator.

In any case (as in the discussion of $\S 1.3$, suitably dualized), the functor $\Phi_{B}$ admits a left adjoint $\check{\Phi}$ provided the category $C$ has equalizers of certain pairs of maps between powers of the object $B$. (The reader may wish to assume that C is some favorite small-limit complete category, for example, the category of topological spaces, locales, groups, topological groups, frames, or, in fact, virtually any reasonably defined category, and that $B$ is an arbitrarily chosen 
object; for our purposes we will tacitly assume that the category $\mathbf{C}$ admits whatever limit constructions we may need at any moment.) It is then apparent that, for each object $X \in|C|$, the evaluation map $e v_{X}$ admits a canonical factorization

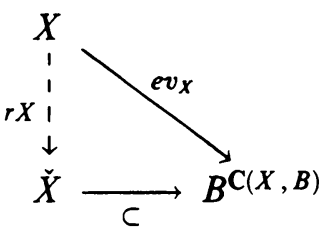

through the object $\check{X}=\check{\Phi} \Phi X$ which (in a manner reminiscent of the dominion construction of Isbell and), employing the universal properties of products, is the simultaneous equalizer of the family of all pairs of maps $(a, b): B^{\mathrm{C}(X, C)} \rightrightarrows$ $B$ satisfying $a \circ e v_{X}=b \circ e v_{X}$. The object $\check{X}$ is called the $B$-dominion of $X$, and the full subcategory of all such is denoted by $\operatorname{dom}_{B} \subset \mathrm{C}$.

Remark. In general, by iterating this process, that is, upon considering the comparison functor for the triple in $\mathbf{S e t}^{\mathbb{B}}$ induced by the adjunction $\check{\Phi} \dashv \Phi$, constructing dominions, etc., while passing to limits (assuming, of course, the availability of these constructions in the category C) at limit ordinal stages, one arrives at the description of the reflective hull of $B$ in $\mathbf{C}$ which is latent in the so-called tower of triples construction of Applegate and Tierney [AT]. It is our goal here to characterize the situation in which this process of constructing dominions terminates (in the precise way indicated in the theorem below) after only one stage.

We say a morphism $X \stackrel{f}{\rightarrow} Y$ in $C$ is $B$-dense provided that, for every pair of maps $(a, b): Y \rightrightarrows B$, if $a \circ f=b \circ f$, then $a=b$. Again employing the universal properties of products, it is clear that the map $f$ is $B$-dense if and only if it is epimorphic with respect to pairs of maps to any set-indexed power of the object $B$, that is, with respect to maps to any object in $|\operatorname{Im}(F)|$. The results of $\S 2$ now, when specialized to the functor $U=\mathbf{C}(-, B): \mathbf{C}^{\mathrm{op}} \rightarrow$ Set , read as follows:

Theorem 3.2. The following statements are equivalent:

1. The functor $U$ is of subdescent type.

2. The natural transformation $\mathbf{r}$ is idempotent; that is, for each object $X \in$ $\left|\mathbf{d o m}_{B}\right|$, the comparison $X \stackrel{r X}{\rightarrow} \check{X}$ is an isomorphism.

3. The maps $\{X \stackrel{r X}{\rightarrow} \check{X}: X \in|\mathbf{C}|\}$ constitute a reflection of the category $\mathbf{C}$ into $\operatorname{dom}_{B}$.

4. The object $B$ is a regular cogenerator for the category $\operatorname{dom}_{B}$.

5. For each object $X \in|\mathbf{C}|$, the map $X \stackrel{r X}{\rightarrow} \check{X}$ is B-dense.

Remark. Since the reflective hull of the object $B$ in $\mathbf{C}$ (were it to exist) must in any case be closed under the formation of products and equalizers, it is clear, given that the equivalent conditions of the theorem hold, that $\mathbf{d o m}_{B}$ is this reflective hull and, moreover, that the comparison functor then serves, upon restriction, to provide a full and faithful (contravariant) representation of dom $_{B}$ as a reflective subcategory of the category of algebras $\mathbf{S e t}^{\mathbf{B}}$. 


\section{EXAMPLES}

4.1. Rigid objects. Provided the category $C$ satisfies some rather mild condition (it is sufficient that, for each noninitial object $X \in|\mathbf{C}|$, the unique map $X \rightarrow 1$ is an epimorphism) and the object $B$ has at least two distinct points and is rigid, which is to say, for every $\alpha \in \mid$ Set $\mid$, each $\alpha$-ary operation $B^{\alpha} \rightarrow B$ is either constant or a projection, the functor $\mathbf{C}(-, B): \mathbf{C}^{\text {op }} \rightarrow$ Set is of subdescent type.

4.2. Groups. Where $\mathbf{C}=\mathbf{G p}$ is the category of groups, the objects (i) $\mathbb{Z}_{n}$, (ii) $\mathbb{Q}$, and (iii) $\mathbb{Q} / \mathbb{Z}$ give rise to contravariant hom-functors $\mathbf{G p}^{\text {op }} \rightarrow$ Set of subdescent type, with categories of dominions (i) abelian groups of bounded exponent $n$, (ii) torsion-free, divisible abelian groups, and (iii) the category of all abelian groups, respectively.

As of this writing, the case in which the interesting group is the integers $\mathbb{Z}$ remains a complete mystery to this author. Here it is clearly useful to consider the class of groups $\mathfrak{K}$ consisting of all subgroups $K \subset \mathbb{Z}^{\alpha}$ (for arbitrary powers of $\mathbb{Z}$ ) which are arbitrary intersections of kernels of group homomorphisms $\mathbb{Z}^{\alpha} \rightarrow \mathbb{Z}$. The class $\mathfrak{K}$ certainly contains the class of $\mathbb{Z}$-dominions, and, by Theorem 3.2 , the functor $\mathbf{G p}(-, \mathbb{Z})$ is of subdescent type if and only if each group $K \in|\mathfrak{K}|$ satisfies $K \cong \check{K}$, which is to say if and only if every group $K$, which is an intersection of such kernels in some power of $\mathbb{Z}$, is an intersection of such kernels in the canonical embedding $e v_{K}: K \rightarrow \mathbb{Z}^{\mathrm{Gp}(K, \mathbb{Z})}$. It seems encouraging to note that, since the group $\mathbb{Z}$ is projective, every finite intersection of kernels (of group homomorphisms to $\mathbb{Z}$ ) in a power of $\mathbb{Z}$ is (a direct summand and, therefore) again isomorphic to a power of $\mathbb{Z}$. On the other hand, it is probably safe to say that infinite powers of $\mathbb{Z}$ are well known for their tendency to exhibit pathological behavior, and indeed, if the equivalent conditions cited above in fact do not hold, it would represent the first such counterexample arising from a well-studied object in a familiar category known to the author.

Where $\mathbf{C}$ is the category of topological groups and continuous group homomorphisms and the object $B$ is the circle group, Set ${ }^{\mathbf{B}}$ is the category of abelian groups, dom $_{B}$ is the category of compact Hausdorff topological groups, and the restriction of the comparison functor to $\mathbf{d o m}_{B}$ is the equivalence of categories well known as Pontryagin duality (compact/discrete case).

4.3. Topological spaces. Let $\mathbf{C}$ be the category of topological spaces and continuous maps, and consider the following table:

\begin{tabular}{|cccc|}
\hline & $B$ & Set $^{\mathbf{B}}$ & dom $_{B}$ \\
\hline 1 & $\mathbf{2}_{d}$ & Boolean algebras & Stone spaces \\
2 & $\mathbb{S}$ & Frames & Sober spaces \\
3 & $\mathbf{2}_{i}$ & CABA & Set \\
4 & {$[0,1]$} & $C^{*}$-Algebras & Compact Hausdorff spaces \\
5 & $\mathbb{R}$ & $C$-Algebras & Real-compact spaces \\
\hline
\end{tabular}

Exhibited are well-known examples of objects $B \in|C|$ for which the functor $U=\mathbf{C}(-, B): \mathbf{C}^{\mathrm{op}} \rightarrow$ Set is of subdescent type, where $2_{d}, \mathbb{S}, \boldsymbol{2}_{i}$, and CABA denote the two-point discrete space, Sierpinski space, two-point indiscrete space, and the category of complete atomic Boolean algebras, respectively (and some 
liberty has been taken in presenting the category of algebras $\mathbf{S e t}^{\mathbf{B}}$ only up to equivalence and, hopefully, in what is its most familiar conception). The astute reader will have already noticed that, in examples 1,3 , and 4 (as well as in some of the previous examples), the functor $U$ exhibits a stronger descent behavior than merely being of subdescent type; in these examples the restriction of $U$ to the subcategory $\operatorname{dom}_{B} \subset \mathbf{C}$ is in fact tripleable, that is, the restriction of the comparison functor $\Phi_{B}$ to $\operatorname{dom}_{B}$ is not merely full and faithful but also has image the entire category of algebras.

4.4. Locales. Having seen the example above, the reader might be inclined to conjecture that a class of topological spaces $C_{0}$ which is distinguished by some nice separation axiom (like, for instance, complete regularity or zerodimensionality), in addition to some degree of compactness, may be identifiable as the class of $B$-dominions for some object $B$ which serves as a regular cogenerator for the class $C_{0}=\mathbf{d o m}_{B}$. One might also ask the analogous question for the case in which $\mathbf{C}$ is the category of locales, and perhaps this is preferable, primarily, since it has been shown [M, J2] that relative to the category of locales the $\kappa$-Lindelöf property, when taken with certain nice separation axioms, is reflective and, secondly, since the results about the category of locales (which are briefly outlined below) generally specialize (upon taking spatial parts) to the best possible analogous results for the category of topological spaces.

In [J2] the epireflective hull $\mathfrak{B}$ of an object $B \in|\mathbf{L o c}|$ is called a nice separation axiom provided it enjoys certain properties which are natural generalizations of properties possessed by the classes of completely regular and zerodimensional locales. It is then shown that, for each (large enough) uncountable regular cardinal $\kappa$, there exists an object $B_{\kappa} \in|\mathfrak{B}|$ such that

(i) Loc $\left(-, B_{\kappa}\right):$ Loc $^{\text {op }} \rightarrow$ Set is of subdescent type and, moreover,

(ii) an arbitrary locale $X$ is a $B_{\kappa}$-dominion if and only if $X$ is a $\kappa$-Lindelöf locale satisfying $X \in|\mathfrak{B}|$.

\section{ACKNOWLEDGMENT}

The author expresses gratitude to A. Hager and W. W. Comfort of Wesleyan University, Middletown, Connecticut; J. R. Hasfura, now of Trinity University, San Antonio, Texas; A. Molitor, now of New Mexico State University, Las Cruces; V. Trnková of Charles University, Prague, for their insightful comments, and, especially, F. E. J. Linton of Wesleyan University, without whose kind and patient advice this paper would not have been possible.

\section{REFERENCES}

[AT] H. Applegate and M. Tierney, Iterated cotriples, Lecture Notes in Math., vol. 137, SpringerVerlag, New York, 1970, pp. 56-99.

[BW] M. Barr and C. Wells, Toposes, triples and theories, Grundlehren Math. Wiss., vol. 278, Springer-Verlag, New York, 1985, pp. 104-112.

[Be] J. Beck, Triples, algebras, and cohomology, Ph.D. Thesis, Columbia Univ., New York, NY, 1967.

[I1] J. R. Isbell, Epimorphisms and dominions, Proc. Confer. Categorical Algebra (La Jolla, 1965), Springer, Berlin, 1966, pp. 232-246.

[I2] — General functorial semantics, Amer. J. Math. 94 (1972), 535-596. 
[J1] P. B. Johnson, $\kappa$-Lindelöf locales and their spatial parts, Cahiers Topologie Géom. Différentielle Catégoriques XXXII-4 (1991), 297-313.

[J2] __ Nice separation axioms, Applied Categorical Structures (to appear).

[L] F. E. J. Linton, Applied functorial semantics. II, Lecture Notes in Math., vol. 80, SpringerVerlag, New York, 1969, pp. 53-74.

[M] J. Madden, $\kappa$-frames, J. Pure Appl. Algebra 70 (1991), 107-127.

[MV] J. Madden and J. Vermeer, Lindelöf locales and realcompactness, Math. Proc. Cambridge Philos. Soc. 99 (1986), 473-480.

Matematický Ústav, Univerzity Karlovy, Sokolovská 83, 18600 Praha 8, Czech RePUBLIC

E-mail address: johnsonecspguk11.bitnet 